\title{
Sur la présence simultanée du cysticercoïde d'un Cestode (Cyclophyllidae, Hymenolepididae) et de la métacercaire d'un Trématode (Dicrocoeliidae) chez Oxydesmus granulosus Palisot de Beauvoir (Myriapode, Diplopode, Polidesmidae) du Dahomey
}

\author{
par C. GABRION, C. GASC et R. ORMIERES \\ Laboratoire de Parasitologie comparée, U.S.T.L., F 34060 Montpellier Cedex \\ Laboratoire de Zoologie I, U.S.T.L., F 34060 Montpellier Cedex
}

\section{Résumé.}

L'examen d'Oxydesmus granulosus Palisot de Beauvoir (Myriapode, Diplopode, Polydesmidae) récoltés à Porto-Novo (Dahomey) nous a permis d'élucider le cycle biologique d'un Cestode, Hymenolepis gilloni (Hunkeler, 1972), parasite à l'état adulte de cinq espèces de Crocidures africaines. Nous avons noté, d'autre part, chez un Oxydesmus la présence de métacercaires d'un Trématode que nous rappoitons, provisoirement, aux Dicrocoeliidae.

\section{Summary.}

On the presence in Oxydesmus granulosus Palisot de Beauvoir (Myriapodes, Diplopodes, Polydesmidae) from the Dahomey, simultaneously of a Cestoda cysticercoid (Cyclophyllidae, Hymenolepididae) and a Trematoda metacercaria (Dicrocoeliidae).

We report observations of cysticercoids of Hymenolepis gilloni (Hunkeler, 1972), a flatworm parasite of five species of Crocidura in Africa.

The cysticercoids are found up against the outside of the gut of a Myriapode, Oxydesmus granulosus, from Porto Novo (Dahomey). We also found the metacercaria of a Trematode which is probably a Dicrocoeliidae. 
En 1957, Baer écrivait à propos de l'étude des parasites de Micromammifères africains : «Il est probable que la recherche méthodique d'Helminthes chez les petits Mammifères d'Afrique réservera encore bien des découvertes intéressantes. De telles recherches ne sauraient être livrées au hasard comme c'est le plus souvent le cas, mais devraient faire l'objet de recherches à la fois du point de vue statistique et du point de vue écologique. »

Les travaux de Baer et Hunkeler en Côte-d'Ivoire répondent à ces préoccupations et permettent de cerner, avec précision, dans un cadre bien défini, la répartition des Helminthes à l'intérieur des populations de Micromammifères.

La recherche, dans les mêmes biotopes des hôtes intermédiaires devait constituer l'étape suivante dans la connaissance du parasitisme de ces animaux. La découverte des stades larvaires d'un Cestode et d'un Trématode chez Oxydesmus granulosus Palisot de Beauvoir (Myriapode, Diplopode, Polydesmidae) au Dahomey montre l'intérêt d'une telle recherche.

O. granulosus (fig. 3,1) est un Diplopode de grande taille (de 4 à $8 \mathrm{~cm}$ ), que l'on rencontre toute l'année, dans les biotopes humides les plus variés, aussi bien au Dahomey que dans les autres pays d'Afrique. On le trouve le plus souvent dans les sous-bois, sous des tapis de feuilles en décomposition.

En juin 1974, nous avons disséqué 14 Oxydesmus provenant du Jardin des Plantes de Porto-Novo (Dahomey). Les dissections se sont étalées sur tout le mois. Parmi les individus examinés, 12 d'entre eux présentaient des formes larvaires d'un Cestode. Ces larves étaient toutes au même stade de développement. Nous avons recueilli au total une centaine de cysticercoïdes, chaque Myriapode infesté présentant entre 10 et 15 métacestodes.

Les cysticercoïdes étaient accolés à la séreuse du tube digestif moyen de l'hôte, parfois isolés, le plus souvent réunis en une masse compacte par une substance amorphe. Le scolex était toujours invaginé dans le cysticercoïde.

Le développement avancé des métacestodes ne nous a pas permis de déterminer s'il s'agit de cysticercoïdes polycéphales comme ceux décrits par Villot (1877) chez des Glomeris sous le nom de Staphylocystis biliarus, ou bien d'une infestation multiple.

A ce stade du développement, les cysticercoïdes (fig. 1 et fig. 3,2) ont un corps globuleux de $300 \mu$ de diamètre, prolongé par un cercomère. Au pôle antérieur les lèvres de l'invagination font légèrement saillie, les corpuszules calcaires sont peu nombreux et groupés aux deux pôles.

La couche externe du tégument, anhyste, est mince. La cavité du cysticercoîde est occupée par le scolex, volumineux $(200 \mu$ de diamètre) caractérisé par un rostre de forme conique (fig. 3,3).

Le scolex (fig. 1 et fig. 3,3) est muni de 4 ventouses inermes mesurant entre 58 et $78 \mu$ de diamètre. Le rostre de $49 \mu$, qui présente une constriction au niveau des ventouses, est armé d'une couronne de 15 crochets (fig. 4), longs de 23 à $27 \mu$. Les crochets sont caractérisés par une garde aplatie en spatule, de 6 à $7 \mu$ de long et 


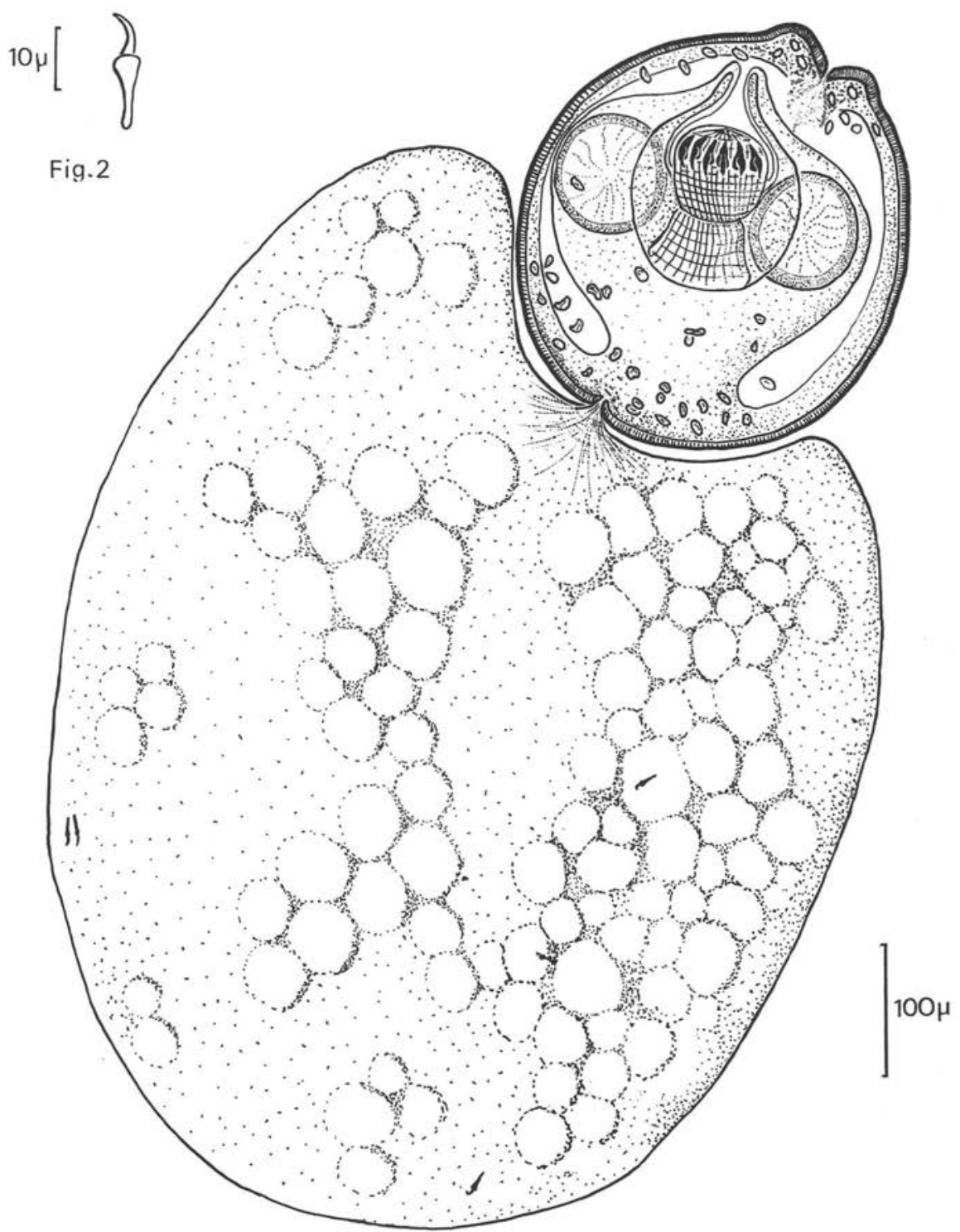

FIG. 1. - Cysticercoïde d'Hymenolepis gilloni Hunkeler, 1972; Fig. 2. - Crochet de l'oncosphère. 
$2 \mu$ de large, surmontée d'une lame de 10 à $12 \mu$. Le manche, légèrement incurvé mesure 9 à $10 \mu$.

Nous n'avons pas noté de variations dans les dimensions des différents éléments du scolex, en particulier, dans la taille des crochets. Le cercomère enveloppe le corps du cysticercoïde dans sa partie postérieure et se prolonge en une expansion grossièremen: hémisphérique de $700 \mu$ de diamètre.

Les crochets de l'oncosphère (fig. 2) longs de $17 \mu$ en moyenne, encore présents, sont disséminés dans le parenchyme du cercomère.

Par la forme, le nombre et les dimensions de ses crochets, le cysticercoïde que nous décrivons s'identifie, sans aucun doute possible à Hymenolepis gilloni décrit par Hunkeler (1972) en Côte-d'Ivoire, chez Crocidura poensis Dallman et signalé par Baer (1972), toujours en Côte-d'Ivoire, chez 4 autres espèces de Crocidures, C. bottegi, C. flavescens, $C$. theresa et un Crocidure dont l'espèce n'a pu être identifiée.

Afin de compléter l'étude de ce cycle, nous avons essayé de réaliser expérimentalement le passage de l'hôte intermédiaire à l'hôte définitif.

Le 29 avril 1974, nous avons fait ingérer avec la nourriture, des cysticercoïdes à une souris blanche. Celle-ci à la dissection, le $1^{\text {er }}$ juillet 1974 ne présentait pas d'Hymenolepis.

Le 5 juillet 1974, nous avons mis en présence une Crocidure C. russula Hermann et un Oxydesmus. Malgré un jeûne prolongé, la Crocidure n'a pas tenté d'ingérer le Myriapode. Ce manque d'intérêt pour l'Oxydesmus pourrait être dû à l'aspect inhabituel de l'animal ou à un phénomène de répulsion provoqué par la substance acide que dégage le Myriapode.

Le 6 juillet 1974, nous avons mêlé, à la nourriture de la Crocidure, une trentaine de cysticercoïdes. Le 8 juillet 1974, nous avons renouvelé l'infestation avec un nombre équivalent de cysticercoïdes. La dissection de l'animal le 13 juillet 1974 n'a pas révélé la présence de Cestodes.

Aucun travail n'a été réalisé sur la systématique des Micromammifères du Dahomey et nous ne savons pas si les espèces de Crocidures chez leqquelles $H$. gilloni a été signalé en Côte-d'Ivoire, existent dans cette région. C'est donc vers la recherche de ces Crocidures et de leurs parasites que se porteront nos investigations.

Sur les 14 Oxydemus disséqués, l'un d'eux présentait 6 métacercaires d'un Trématode. Elles étaient logées dans la musculature de la plaque collaire, immédiatement après la tête.

Nous n'avons pu isoler les métacercaires de la musculature. Les mauvaises conditions dans lesquelles ont été réalisées ces observations ne nous ont pas permis d'étudier les systèmes excréteur et sensoriel. Seuls, les caractères morphologiques les plus apparents ont pu être notés.

Les métacercaires paraissent dépourvues de kyste métacercarien. Le corps de l'animal, arrondi aux deux extrémités mesure $0,34 \mathrm{~mm}$ de longueur et $0,18 \mathrm{~mm}$ de largeur, au niveau de l'acétabulum. Les ventouses, sensiblement de la même taille ont un diamètre de $60 \mu$. La ventouse orale est armée d'un stylet de $32 \mu$ (fig. 3-4). 

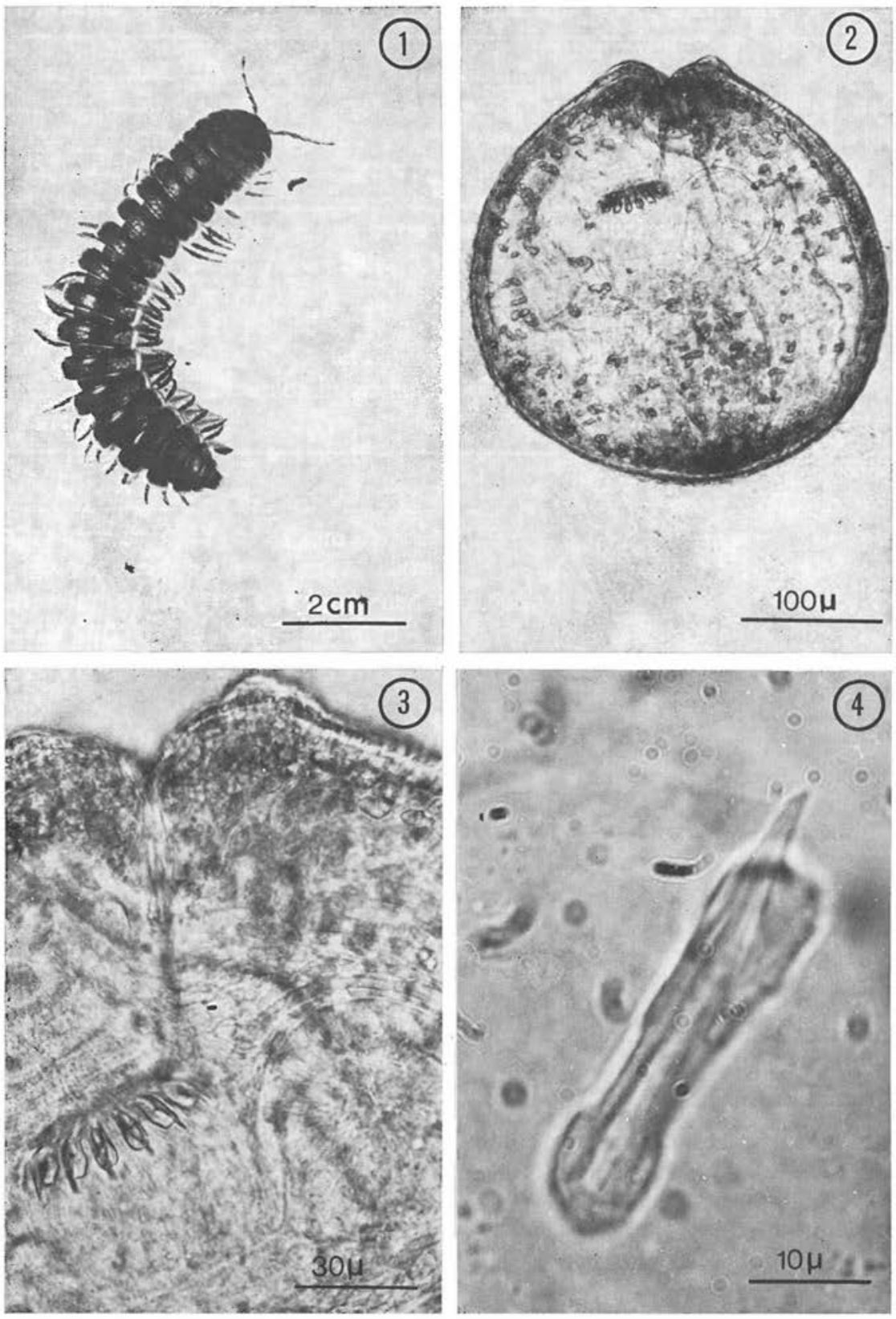

Fig. 3. - 1. Oxydesmus granulosus Palisot de Beauvoir; 2. Micropho ographie du cysticercoïde d'Hymenolepis gilloni. Le scolex invaginé est bien visible ; 3. Détails du cysticercoïde d'H. gilloni. On observe la forme du rostre et, au centre, 6 crochets rostraux; 4. Microphotographie du stylet de la métacercaire observée chez $O$. granulosus. 
Celui-ci présente un manche étroit de $4 \mu$, élargi aux deux extrémités. A la partie postérieure arrondie, large de $6 \mu$ s'insèrent les muscles. La pointe du stylet, longue de $12 \mu$, se projette en avant de l'expansion antérieure.

La morphologie du stylet nous permet de rapporter ces métacercaires à un Dicrocoeliide.

Nous avons recherché les espèces de Dicrocoeliides signalées en Afrique Occidentale (Tableau I) Baer (1971) nous maintenons dans cette famille les Brachycoeliinae qui en avaient été séparés par Cheng (1960).

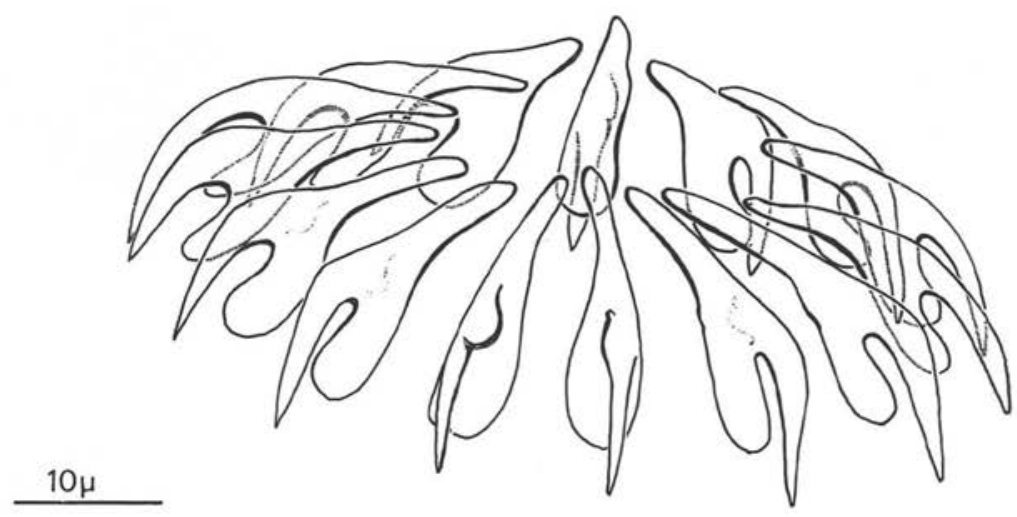

FIG. 4. - Couronne de crochets du cysticercoïde.

Les caractères morphologiques (persistance du stylet) rapprocheraient la métacercaire de celle de Mesocoelium monas obtenue par Thomas (1965) chez deux espèces de Fourmis. Cependant, cette métacercaire enkystée, possède un stylet apparemment différent de celui que nous avons observé.

La présence simultanée de métacercaires et de cysticercoïdes d'Hymenolepis gilloni chez l'Oxydesmus rend séduisante l'idée d'un même hôte définitif pour les deux Helminthes. Dans ce cas, les métacercaires pourraient être celles d'Opistholecithum margaritae signalées chez deux espèces de Crocidures de Côte-d'Ivoire, $C$. flavescens et C. poensis, hôtes habituels d' $H$. gilloni.

Néanmoins, il est difficile, à partir des seuls éléments en notre possession, d'affirmer l'appartenance des métacercaires à l'une quelconque de ces espèces. D'autre part, l'inventaire des Helminthes de Vertébrés terrestres, dans ces régions étant incomplet l'appartenance des stades larvaires observés à une espèce de Dicrocoeliide non encore identifiée n'est pas à exclure.

Enfin, la présence du stylet et le manque de kyste métacercarien pourraient être le signe d'une infestation accidentelle, l'Oxydesmus n'étant pas, pour la métacercaire l'hôte intermédiaire habituel. 


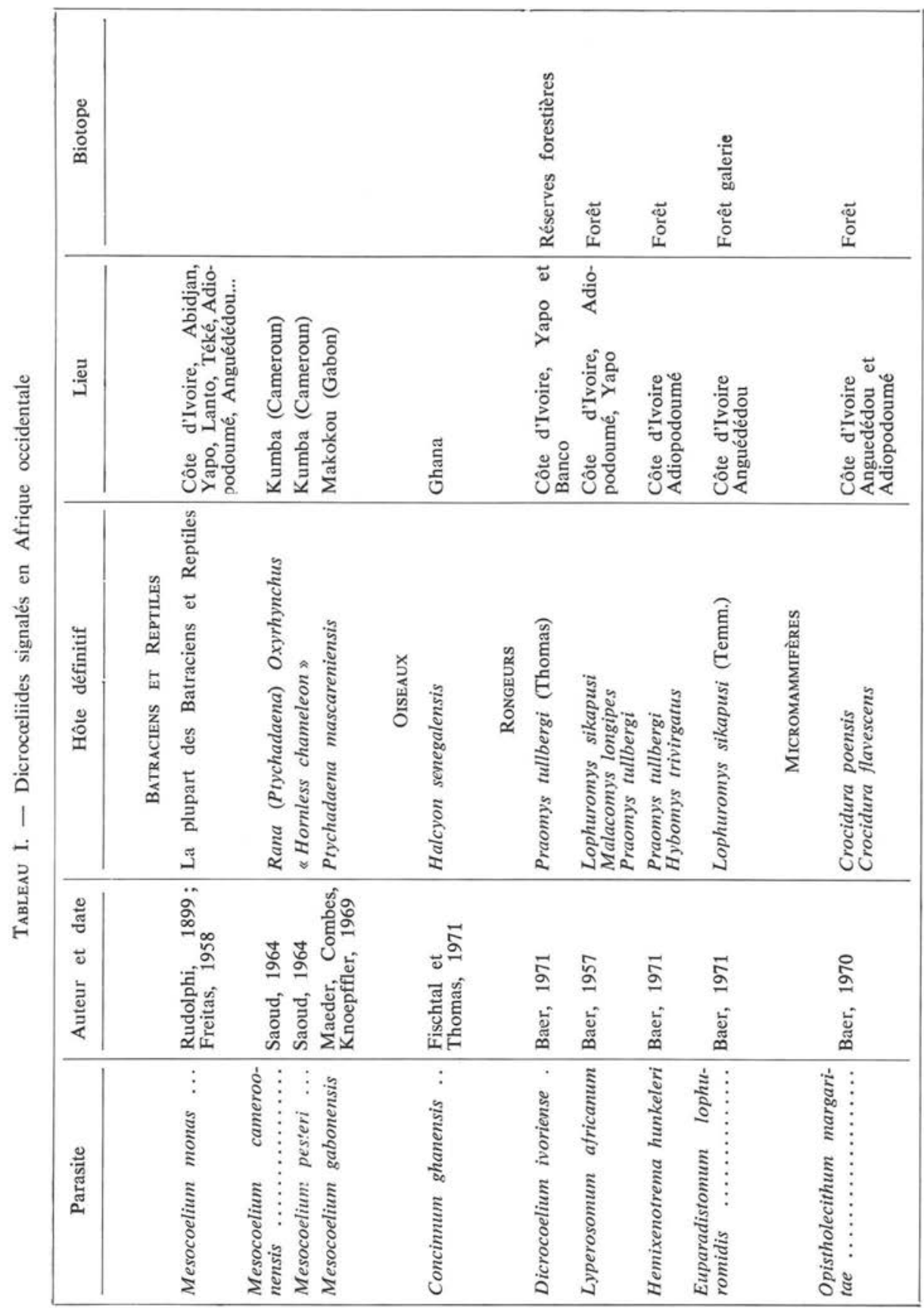




\section{Conclusions.}

Plusieurs Cestodes signalés chez les Insectivores africains avaient été assimilés. lors de leur découverte à des Cestodes d'Insectivores d'Europe. Hunkeler (1974), dans son travail sur les Cestodes de Micromammifères d'Afrique, s'appuyant sur des différences morphologiques des adultes, considère que l'Helminthofaune des Crocidures d'Europe et celle des Crocidures d'Afrique sont différentes. C'est ainsi qu'il distingue $H$. gilloni, espèce africaine, d'H. scalaris, l'espèce européenne la plus proche.

La découverte du cysticercoïde d'H. gilloni chez un Myriapode, venant après celle du cysticercoïde d'H. scalaris chez un Coléoptère carabique Pterostichus sp. (Jourdane, 1972 ) et les caractères des métacestodes confirment l'originalité de $H$. gilloni par rapport à $H$. scararis.

La présence de métacercaires de Dicrocoeliidae, chez un Myriapode Diplopode est tout aussi intéressante. En effet, cette découverte venant après celle de métacercaires d'un Brachylecithum chez Phalangium opilio en France (Gabrion et Ormières, 1973) confirme l'intérêt qu'il y aurait à orienter la recherche des métacercaires de ces Trématodes vers ces groupes d'Arthropodes.

\section{Bibliographie}

BaER (J.-G.), 1957. - Trématodes et Cestodes récoltés en Côte d'Ivoire, avec remarques sur la famille des Dicrocoeliidae, Odhner et sur les parasites des Damans. Rev. Suisse Zool., 64, 547-575.

BAer (J.-G.), 1971. - Trématodes de Rongeurs récoltés en Côte d’Ivoire. Z. Parasitenk., $37,226-254$.

Baer (J.-G.), 1970. - Deux Trématodes nouveaux de Musaraignes d'Afrique occidentale. Ann. Inst. Biol. Univ. Nal. Autón. Mexico, 41, 11-18.

BAer (J.-G.), 1972. - Liste critique des parasites (Monogènes, Cestodes et Trématodes) et de leurs hôtes en République de Côte d'Tvoire. Acta Tropica, 29, 341-361.

Cheng (T.C.), 1959. - Studies on the Trematode family Brachycoeliidae, II. Revision of the Genera Glypthelmins (Stafford, 1900), Stafford, 1905 and Margeana (Cort, 1919); and the description of Renoldstrema n. gen. (Glypthelminae, n. subfam.). Amer. Midl. Nat., 51, 68-88.

CHENG (T. C.), 1960. - Studies on the Trematode family Brachycoeliinae. IV. A revision of the genus Mesocoelium (Odhner, 1911), and the status of Pintnaria (Poche, 1907). Amer. Midl. Nat., 63, 439-469.

Fischthal (J. H.) et Thomas (J. D.), 1971. - Two Dicrocoeliid trematodes from Ghana : Concinnum ghanensis n. sp. and Dicrocoelium hospes (Looss, 1907). J. Helminth, $45,337-340$.

Gabrion (C.) et Ormit̀res (R.), 1973. - Présence chez Phalangium opilio L. (Arachnida, Phalangidae) de métacercaires d'un trématode du genre Brachylecithum (Ström, 1940) (Dicrocoeliidae). C.R. Acad. Sci. (Paris), 276, 2957-2959. 
HunKeler (P.), 1973. - Les Cestodes parasites des petits mammifères (Rongeurs et Insectivores) de Côte d'Ivoire et de Haute-Volta. Rev. Suisse Zool., 80, 811-930.

JouRdANe (J.), 1972. - Découverte du cysticercö̈de d'Hymenolepis scalaris (Dujardin, 1845). Appartenance systématique de Staphylocystis biliarus (Villot, 1877). Ann. Parasit. hum. comp., 47, 289-303.

Maeder (A. M.), Combes (C.) et Knoepffler (L. Ph.), 1969. - Parasites d'Amphibiens du Gabon: Mesocoeliidae (Digenea). Taxonomie et chorologie du genre Mesocoelium (Odhner, 1911). Biologica Gabonica, 4, 289-303.

SAOud (F.A.), 1964. - On some digenetic trematodes (Mesocoelium sp.) from Amphibia and Reptilia of the Cameroons. J. Helminth., 38, 291-302.

Thomas (J.D.), 1965. - The anatomy life history and size allometry of Mesocoelium monodi. J. Zool., 146, 413-446.

Villot (A.), 1877. - Sur une nouvelle forme larvaire des Cestoides. C.R. Acad. Sci. (Paris), 84, 1097. 